\title{
Emerging Antibacterial Coated Dental Implants: A Preventive Measure for Peri-implantitis
}

\author{
${ }^{1}$ Varun Yarramaneni, ${ }^{2}$ IN Aparna, ${ }^{3}$ Akanksha Sachdeva, ${ }^{4}$ Dhanasekar Balakrishnan, ${ }^{5}$ Nayana Prabhu
}

\section{ABSTRACT}

Dental implants are the modern marvel and are widely accepted as a reconstructive treatment modality for tooth replacement.

In recent times, there has been a marked progress in the clinical success rates of dental implants, but implant failures as a result of infections are continuing at an alarming rate of $8 \%$ per year, translating into 1 million failures worldwide.

Perimucositis and peri-implantitis are the chief complications reported postimplant surgery that effects its short- and long-term success. Peri-implantitis is characterized by clinical and radiological bone loss around the implant accompanied with an inflammatory reaction of the peri-implant mucosa and is an irreversible condition, whereas perimucositis is a reversible inflammatory change.

Implant surfaces provide an ideal substrate for bacterial adhesion forming a biofilm. Biofilm performs vast functions ranging from physical defensive barrier against phagocytic predation to working as a selective permeable barrier. This limits the diffusion of systemic antimicrobial agents that are capable of damaging the bacterial complexes. These rapidly growing bacteria give rise to a chronic infection which is difficult to eradicate by conventional antibiotic therapy.

To inhibit peri-implant infections, various functional modifications in the implant surfaces have been suggested. The coatings on the titanium implant are incorporated with disinfectants, antibiotics as well as antimicrobial peptides AMPs.

This paper is an attempt to review all the antibiotic coatings available for a titanium implant and discuss their prospective future to prevent peri-implant infections.

Keywords: Antibiotics, Antimicrobial peptides, Bioactive coatings, Dental implants, Peri-implantitis.

How to cite this article: Yarramaneni V, Aparna IN, Sachdeva A, Balakrishnan D, Prabhu N. Emerging Antibacterial Coated Dental Implants: A Preventive Measure for Peri-implantitis. World J Dent 2016;7(4):195-198.

Source of support: Nil

Conflicts of interest: None

\section{INTRODUCTION}

Titanium and titanium alloys are the most preferred implant materials used in orthopedic and dental clinics due to its excellent biocompatibility, surface characteristics,

\footnotetext{
${ }^{1,3}$ Postgraduate, ${ }^{2}$ Professor, ${ }^{4}$ Professor and Head, ${ }^{5}$ Associate Professor

${ }^{1-5}$ Department of Prosthodontics and Crown and Bridge, Manipal College of Dental Sciences, Manipal, Karnataka, India
}

Corresponding Author: IN Aparna, Professor, Department of Prosthodontics and Crown and Bridge, Manipal College of Dental Sciences, Manipal, Karnataka, India, Phone: +9845648763 e-mail: prostho.manipal@gmail.com and mechanical properties. Long-term clinical efficacy of these implants is influenced by persistent infections at the implant-tissue interface leading to peri-implantitis.

Peri-implantitis has been characterized by an inflammatory process around an implant, which includes both soft tissue inflammation and progressive loss of supporting bone beyond biological bone remodeling., ${ }^{1,2}$

Percutaneous and transmucosal implants, such as external fixation pins and dental implants are even more vulnerable to bacterial contamination. ${ }^{3,4}$ Pathogens, such as Staphylococcus aureus, Staphylococcus epidermidis, and Pseudomonas aeruginosa can be acquired at the time of surgery or at a later stage (e.g., via homogeneous state) $)^{5}$ and can attach to the surface of the implant to nucleate infections.

Implant surfaces provide an ideal substrate for bacterial adhesion forming a biofilm. The biofilm performs vast functions ranging from physical defensive barrier against phagocytic predation to working as a selective permeable barrier. ${ }^{5}$ This limits the diffusion of systemic antimicrobial agents that are capable of damaging the bacterial complexes. Bacterial biofilms are several folds more resistant to antibiotics compared to their planktonic state. ${ }^{6}$

These rapidly growing bacteria give rise to a chronic infection which is difficult to eradicate by conventional antibiotic therapy. Continuous exposure of bacteria to high doses of conventional antimicrobials exerts a selective pressure on these microorganisms, positively affecting their ability to resist broad spectrum antibiotics. ${ }^{7}$ Methicillin-resistant Staphylococcus aureus (MRSA) poses a serious threat and can lead to devastating effects in absence of an effective antimicrobial treatment.

Two challenges that are usually faced with the local delivery of antibiotics at the implant site are (a) antibacterial resistance and (b) achieving antimicrobial activity without impairing osseointegration. An alternative treatment would be the usage of nonconventional antibiotics for local drug delivery that do not potentiate the resistant strains. ${ }^{8}$

\section{COATINGS LOADED WITH ANTIBIOTICS}

Systemic antibiotic prophylaxis is a common practice to prevent periprosthetic infection. However, systemic administration of antibiotics has many shortcomings which include relatively low drug concentration at the target site and potential toxicity. Topical application of antibiotics has attracted much attention. Thus, researchers 
are trying to develop antibiotic-loaded coatings on the titanium implants. ${ }^{9}$

These bioactive coatings release antibiotics, such as gentamycin, vancomycin, and amoxicillin in a meticulous manner and require a vector. Calcium phosphates, which are known to be biocompatible and osteoconductive, have been verified to be potential vectors of bioactive molecules, but antibiotics cannot be incorporated during its formation because of high processing temperature. This limits the adsorption of these drugs onto the surface and affects its release. Zhao et $\mathrm{al}^{9}$ studied and concluded that antibiotic-loaded hydroxyapatite (HA) coatings on titanium boosts infection prophylaxis compared with standard HA coatings.

Various methods of physical adsorption have been reported: (a) Loading by dipping method leads to burst release of antibiotics; (b) application of a lipid layer serves as hydrophobic barrier can retard the drug release; (c) biomimetic method by immersion into a supersaturated solution of calcium phosphate; (d) controlled release of antibiotics by biodegradable polymers and sol-gel coatings; and (e) electrospray deposition of amoxicillin combined with poly(lactic-co-glycolic acid) (PLGA).

A new biodegradable gentamicin-loaded Poly D, L, Lactic acid coating has been developed by Lucke et al to prevent implant-related osteomyelitis in rats. An optimized multilayered vancomycin-incorporated silica sol-gel film shows zero release of vancomycin up to 2 weeks. ${ }^{10,11}$

Vancomycin covalently bonded to titanium using a solid-state synthesis preserves the activity of the antibiotic. It is a preferred antibiotic for protection against both bacterial adhesion and biofilm formation by S. epidermidis. Titanium surface tethered with vancomycin is biocidal to the bacterial cell wall. It is hypothesized that Lys-D-Ala-D-Ala peptidoglycan reversibly cross links with the cell wall that results in release of bacterial remnants leaving the surface bound vancomycin free to engage other bacteria. Vancomycin-titanium surface reportedly has minimal effect on Escherichia coli. Clinically, it displays low musculoskeletal toxicity. ${ }^{12,13}$

Gentamycin is a thermostable, broad-spectrum antibiotic (aminoglycoside) that is widely used for coating titanium implants. Loading of gentamycin into the nanotubes is effective in minimizing initial bacterial adhesion without adverse influence on the good cytocompatibility of the nanotubes. However, elution of gentamycin is still too fast with all the drugs delivered within 50 to 150 minutes in phosphate-buffered saline. ${ }^{14}$

There are various limitations of these antibacterial loaded coatings. Firstly, the potential drug resistance attained by bacterial phenotypes. Secondly, inability to produce an antibacterial-laden coating with a long delivery time at effective concentrations. Thirdly, some drug carriers release antibiotics at concentrations lower than the minimum inhibitory concentration for an indefinite period. ${ }^{9}$

\section{ORGANIC NONANTIBIOTIC ANTIMICROBIAL COATINGS}

\section{Antimicrobial Peptides}

The use of antimicrobial peptides (AMPs) as an antimicrobial approach have been introduced due to their broad-spectrum activity against bacteria, fungi and virus, low host cytotoxicity, and low bacterial resistance. ${ }^{15}$ These AMPs are derived from human proteins which are physically adsorbed or covalently attached on to the implant surface. ${ }^{16-18}$

Pathogenic bacteria are less likely to develop resistance against AMPs because of the inability to redesign their membrane due to limited lipid synthesis capability. These AMPs cause membrane lysis either by barrel stave, toroidal pore, or carpet mechanisms, although no single mechanism can be defined for all peptides. ${ }^{19,20}$

GL13K is a small cationic AMP, i.e., derived from human salivary protein, parotid secretory protein (HPSP). Balhara et $\mathrm{al}^{6}$ in 2013, and Chen et $\mathrm{al}^{18}$ in 2014, found that GL13K has strong anti-inflammatory and antibacterial activities against both Gram-negative and biofilm-forming bacteria particularly effective against Pseudomonas aerogenosa. GL13K peptide apart from bactericidal effect has additional resistance through hydrolytic and mechanical changes with no significant release of peptides from the titanium surface. Holmberg et $\mathrm{al}^{21}$ found GL13K to be cytocompatible with osteoblasts and human gingival fibroblasts.

To produce GL13K, the GL13NH2 peptide was modified by introducing three lysine residues which switch the activities from agglutination to bactericidal. ${ }^{22}$ GL13K interacts with artificial membranes in $\beta$ sheet conformations to produce membrane holes. ${ }^{6}$ Significant antimicrobial activity against Gram-negative bacterium Porphyromonas gingivalis has been seen when GL13K peptide is bonded to titanium surfaces using silane coupling agents. ${ }^{21}$ It was seen that the activity of GL13K rapidly diminished when exposed to biofilm which could be solved by introduction of D-amino acid version, i.e., D-GL13K. Long-term stability and clinical effectiveness of GL13K needs to be future researched.

Chlorhexidine, chloroxylenol, and poly(hexamethylene biguanide) are good organic antimicrobial alternatives. Chlorhexidine is well known for its immense application for the treatment of periodontal infections and as a mouthwash. ${ }^{23}$ Chlorhexidine is adsorbed onto the surface oxide layer on the titanium implant by inducing surface mineralization with HA coating, spray depositing polylactide coating on anodized surface, and impregnation of Poly MethylMethAcrylate (PMMA) and calcium phosphate coating. It has a large inhibition zone for $S$. aureus. 
Recently, essential oils are used to fabricate thin film coatings. They are derived from nonsynthetic terpinen4-ol, a major constituent of Melaleuca altenifolia, and have a broad spectrum antibacterial, antiviral, antifungal, and anti-inflammatory activity. ${ }^{24}$

\section{Bioactive Antibodies}

Antibodies or immunoglobulins have the intrinsic capacity to opsonize microbes and phagocyte them, thereby reducing the virulence. It is a natural immune function of the body which is critically exploited at the implant and wound sites. These antibodies operate independently of antibiotic resistance mechanisms. The most significant antibody subtype is immunoglobulin G (IgG). The release of commercially pooled human polyclonal IgG from hydrophilic polyurethane (PU) hydrogel has shown validated results against the clinical strain of E. coli. ${ }^{25}$

\section{INORGANIC NONANTIBIOTIC ANTIMICROBIAL COATINGS}

It has been observed that a thin layer of HA coatings on titanium (Ti) implant surface can be deposited by a mechanism known as "magnetron sputtering." It is an expedient process that allows the mechanical properties of Ti to be preserved as well as maintains the bioactivity of coated HA.

Similar to HA, silver $(\mathrm{Ag})$ too is used to dope $\mathrm{Ti}$ implants for antibacterial applications. It has a broad, long-lasting activity against both Gram-positive and Gramnegative bacteria.

The potency of $\mathrm{Ag}$ as an antibacterial coating was suggested to be dependent on its biological active form, soluble Ag ions or Ag clusters, to interfere with the integrity of the bacterial cells and to bind to the enzymes and proteins within the bacteria. ${ }^{26,27}$ Silver doping can effectively inhibit bacterial adhesion and growth without compromising the activity of osteoblasts and epithelial cells. ${ }^{28}$ In vivo studies have demonstrated that Ag coatings exhibit no local or systemic side effects.

Silver can be introduced by various techniques, such as magnetron sputtering, plasma immersion ion implantation (PIII), pulsed filtered cathodic vacuum arc deposition, physical vapour deposition (PVD), and many more. ${ }^{28}$ It is believed that anodization of $\mathrm{Ag}$ can yield extra antibacterial activity which is of special interest for dental implants. ${ }^{9}$

Recently, nanosilver cement has also been proved to have high antibacterial activity against methicillinresistant S. Epidermidis (MRSE) and MRSA. ${ }^{28,29}$ Besides silver, other inorganic agents, such as copper, fluorine, nitrogen, calcium, and zinc have been introduced as potential antimicrobial agents.
Tantalum alloys are known to have excellent biocompatibility when used as a protective coating. Recently, it was reported that Ag-doped TaN and Cu-doped TaN with nanoparticles can decrease the multiplication of E. coli bacteria. A twin-gun magnetron sputtering system is used for deposition of TaN-Ag coatings. TaN and TaN-Ag coated Ti possessed higher optical density value and showed better Human Gingival Fibroblasts (HGF) cell viability and proliferation than the uncoated sample. ${ }^{30}$

Raulli et $\mathrm{al}^{31}$ has verified nitrous oxide (NO) to be antibacterial in a series of solution-based in vitro assays. Exogenic NO may be used to prevent the survival of pathogenic bacteria on the implant surface because of the direct bactericidal effect and/or by augmenting the natural antimicrobial ability of the immune system. ${ }^{9}$ Sol-gel coatings are capable of NO release from implants. The local surface flux of NO generated from these sol-gel materials significantly reduces the adhesion of three common opportunistic pathogens $P$. aeruginosa, S. aureus, and S. epidermidis. The NO-releasing xerogels have also been shown to kill adherent bacteria cells. Although application of NO releasing xerogels on titanium have not been reported, but it is expected to be a viable method. ${ }^{32,33}$

\section{Adhesion Resistant Coatings}

Surface modifications can significantly affect initial adhesion and biofilm formation on the implant surface. A bacterial adhesion resistant surface can by methods like ultraviolet (UV) light radiation, changing the crystalline structure of surface oxide layer, and the use of antiadhesive polymer coatings.

In vitro and in vivo experiments exhibited evident results that UV light pretreatment of Ti substantially enhances its osteoconductive capacity on Ti by progressive elimination of hydrocarbons from the $\mathrm{TiO}_{2}$ surface. $9,34,35$

Polymer coatings, such as poly(methylmethacrylic acid) and polyethylene glycol are known to significantly reduce the adhesion of $S$. aureus and S. epidermidis. ${ }^{9}$

Bioactive molecules like sericin, arginine glycine aspartic acid Arginylglycylaspartic acid (RGD), chitosan, and hyaluronic acid possess antibacterial activity.

\section{CONCLUSION}

Bacterial infection postimplant placement is a serious complication. To reduce the periprosthetic infections, good progress has been made to fabricate coating materials that are biocompatible and are antimicrobial in nature that enhance the clinical success rate of implants. Various localized methods to address the concerns of orthopedic implants are reported, but future studies on dental implants should be explored. Hence, our supreme interest lies in emerging with effective ways of reducing infection. 


\section{REFERENCES}

1. Sanz M, Chapple IL. Clinical research on peri-implant diseases: Consensus report of Working Group 4. J Clin Periodontol 2012 Feb;39(Suppl 12):202-206.

2. Kwan JY, Newkirk SM. Ultrasonic endoscopic periodontal debridement. Minim Invasive Periodont Ther 2014 Nov.

3. Green SA, Ripley MJ. Chronic osteomyelitis in pin tracks. J Bone Joint Surg Am 1984 Sep;66(7):1092-1098.

4. Rimondini L, Valle CD, Cochis A, Azzimonti B, Chiesa R. The biofilm formation onto implants and prosthetic materials may be contrasted using gallium (3+). Key Eng Mater 2013 Nov;587:315-320.

5. Costerton JW, Stewart PS, Greenberg EP. Bacterial biofilms: a common cause of persistent infections. Science 1999 May;284(5418):1318-1322.

6. Balhara V, Schmidt R, Gorr S-U, DeWolf C. Membrane selectivity and biophysical studies of the antimicrobial peptide GL13K. Biochim Biophys Acta 2013 Sep;1828(9):2193-2203.

7. Andersson DI, Hughes D. Persistence of antibiotic resistance in bacterial populations. FEMS Microbiol Rev 2011 Sep;35(5):901-911.

8. Kazemzadeh-Narbat M. Antimicrobial peptides on calcium phosphate-coated titanium for the prevention of implantassociated infections. Biomaterials 2010 Dec;31(36):9519-9526.

9. Zhao L, Chu PK, Zhang Y, Wu Z. Antibacterial coatings on titanium implants. J Biomed Mater Res B Appl Biomater 2009 Oct;91(1):470-480.

10. Lucke M, Schmidmaier G, Sadoni S, Wildemann B, Schiller R, Haas NP, Raschke M. Gentamicin coating of metallic implants reduces implant-related osteomyelitis in rats. Bone 2003 May;32(5):521-531.

11. Radin S, Ducheyne P. Controlled release of vancomycin from thin sol-gel films on titanium alloy fracture plate material. Biomaterials 2007 Mar;28(9):1721-1724.

12. Antoci V Jr, King SB, Jose B, Parvizi J, Zeiger AR, Wickstrom E, Freeman TA, Composto RJ, Ducheyne P, Shapiro IM, et al. Vancomycin covalently bonded to titanium alloy prevents bacterial colonization. J Orthop Res 2007 Jul;25(7):858-866.

13. Antoci V Jr, Adams CS, Parvizi J, Davidson HM, Composto RJ, Freeman TA, Wickstrom E, Ducheyne P, Jungkind D, Shapiro IM, et al. The inhibition of Staphylococcus epidermidis biofilm formation by vancomycin-modified titanium alloy and implications for the treatment of periprosthetic infection. Biomaterials 2008 Dec;29(35):4684-4690.

14. Popat KC, Eltgroth M, Latempa TJ, Grimes CA, Desai TA. Decreased Staphylococcus epidermis adhesion and increased osteoblast functionality on antibiotic-loaded titania nanotubes. Biomaterials 2007 Nov;28(32):4880-4888.

15. Chen, X.; Li, Y.; Aparicio, C. Biofunctional coatings for dental implants. In: Nazarpour, S.; Chaker, M., editors. Thin films and coatings in biology. Biological and medical physics, biomedical engineering series. Springer; 2013. p. 105-143.

16. Kazemzadeh-Narbat M, Lai BF, Ding C, Kizhakkedathu JN, Hancock RE, Wang R. Multilayered coating on titanium for controlled release of antimicrobial peptides for the prevention of implant-associated infections. Biomaterials 2013 Aug;34(24):5969-5977.

17. Gao GZ, Lange D, Hilpert K, Kindrachuk J, Zou YQ, Cheng JT, Kazemzadeh-Narbat M, Yu K, Wang R, Straus SK. The biocompatibility and biofilm resistance of implant coatings based on hydrophilic polymer brushes conjugated with antimicrobial peptides. Biomaterials 2011 Jun;32(16): 3899-3909.
18. Chen X, Hirt H, Li Y, Gorr S-U, Aparicio C. Antimicrobial GL13K peptide coatings killed and ruptured the wall of Streptococcus gordonii and prevented formation and growth of biofilms. PLoS One 2014 Nov;9(11):e111579.

19. Abraham T, Lewis RNAH, Hodges RS, McElhaney RN. Isothermal titration calorimetry studies of the binding of the antimicrobial peptide gramicidin $S$ to phospholipid bilayer membranes. Biochemistry 2005 Feb;44(6):11279-11285.

20. Nguyen LT, Haney EF, Vogel HJ. The expanding scope of antimicrobial peptide structures and their modes of action. Trends Biotechnol 2011 Sep;29(9):464-472.

21. Holmberg KV, Abdolhosseini M, Li Y, Chen X, Gorr SU, Aparicio C. Bioinspired stable antimicrobial peptide coatings for dental applications. Acta Biomater 2013 Sep;9(9): 8224-8231.

22. Gorr S-U, Sotsky JB, Shelar AP, Demuth DR. Design of bacteriaagglutinating peptides derived from parotid secretory protein, a member of the bactericidal/permeability increasing-like protein family. Peptides 2008 Dec;29(12):2118-2127.

23. Campbell AA, Song L, Li XS, Nelson BJ, Bottoni C, Brooks DE, DeJong ES. Development, characterization, and anti-microbial efficacy of hydroxyapatite-chlorhexidine coatings produced by surface-induced mineralization. J Biomed Mater Res 2000;53(4):400-407.

24. Bazaka K, Jacob M, Troung VK, Crawford RJ, Inanova EP. The effect of polyterpenol thin film surfaces on bacterial viability and adhesion. Polymers 2011;3(1):388-404.

25. Rojas IA, Slunt JB, Grainger DW. Polyurethane coatings release bioactive antibodies to reduce bacterial adhesion. J Control Release 2000 Jan;63(1-2):175-189.

26. Schreurs WJ, Rosenberg H. Effect of silver ions on transport and retention of phosphate by Escherichia coli. J Bacteriol 1982 Oct;152(1):7-13.

27. Ghandour W, Hubbard JA, Deistrung J, Hughes MN, Poole RK. The uptake of silver ions by E. coli: Toxic effects and interactions with copper ions. Appl Microbiol Biotechnol 1988;28:559-565.

28. Chen W, Liu Y, Courtney HS, Bettenga M, Agrawal CM, Bumgardner JD, Ong JL. In vitro anti-bacterial and biological properties of magnetron co-sputtered silver-containing hydroxyapatite coating. Biomaterials 2006 Nov;27(32): 5512-5517.

29. Stigter M, de Groot K, Layrolle P. Incorporation of tobramycin into biomimetic hydroxyapatite coating on titanium. Biomaterials 2002 Oct;23(20):4143-4153.

30. Huang H-L, Chang Y-Y, Lai M-C, Lin C-R, Lai C-H, Shieh T-M. Antibacterial TaN-Ag coatings on titanium dental implants. Surf Coat Technol 2010 Nov;205(5):1636-1641.

31. Raulli R, McElhaney-Feser G, Hrabie JA, Cihlar RL. Antimicrobial properties of nitric oxide using diazeniumdiolates as the nitric oxide donor. Recent Res Dev Microbiol 2002;6:177-183.

32. Nablo BJ, Rothrock AR, Schoenfisch MH. Nitric oxidereleasing sol-gels as antibacterial coatings for orthopaedic implants. Biomaterials 2005 Mar;26(8):917-924.

33. Hetrick EM, Schoenfisch MH. Antibacterial nitric oxide releasing xerogels: cell viability and parallel plate flow cell adhesion studies. Biomaterials 2007 Apr;28(11):1948-1956.

34. Aita H, Hori N, Takeuchi M, Suzuki T, Yamada M, Anpo M, Ogawa T. The effect of ultraviolet functionalization of titanium on integration with bone. Biomaterials 2009 Feb;30(6):1015-1025.

35. Samizade S, Kazemian M, Ghorbanzadeh S, Amini P. Periimplant diseases: treatment and management. Int J Contemp Dent Med Rev 2015;2015:Article ID: 070215. 